\title{
Biosynthesis of fraxetin from three different substrates using engineered Escherichia coli
}

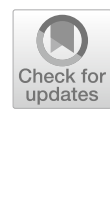

Seung Hoon An, Gyu-Sik Choi and Joong-Hoon Ahn*

\begin{abstract}
Fraxetin, which is a simple coumarin, is a phytochemical present in medicinal plants, such as Fraxinus rhynchophylla, and Cortex Fraxini. In plants, it serves as a controller of iron homeostasis. The health-enhancing activities of fraxetin, such as anticancer, neuroprotective and antibacterial activities, are known. Scopoletin 8-hydroxylase $(\mathrm{S} 8 \mathrm{H})$ is a key enzyme involved in the synthesis of fraxetin from scopoletin. Scopoletin can be synthesized either from esculetin by O-methylation or from ferulic acid by feruloyl CoA $6^{\prime}$-hydroxylase $\left(F \sigma^{\prime} H\right)$ and 4-coumaric acid $C o A$ ligase $(4 C L)$. To enable fraxetin synthesis, the fraxetin biosynthesis pathway was introduced into Escherichia coli. Three distinct routes, from ferulic acid, esculetin, and scopoletin, were designed for the synthesis of fraxetin. In the first approach, E. coli strain harboring $\mathrm{S} 8 \mathrm{H}$ was used and found to synthesize $84.8 \mu \mathrm{M}$ fraxetin from $100 \mu \mathrm{M}$ scopoletin. Two E. coli strains were used for the other two approaches because these approaches required at least two enzymatic reactions. Through this approach, $41.4 \mu \mathrm{M}$ fraxetin was synthesized from $100 \mu \mathrm{M}$ esculetin, while $33.3 \mu \mathrm{M}$ fraxetin was synthesized from $100 \mu \mathrm{M}$ ferulic acid.
\end{abstract}

Keywords: Coumarin, Fraxetin, Scopoletin 8-hydroxylase

\section{Introduction}

Coumarins (benzo-alpha-pyrones) were first isolated from the tonka bean (Dipteryx odorata) in 1820. Since then, their presence has been detected in various parts of different plants, including the fruit (e.g., in Bael fruit or Aegle marmelos), seed (e.g., in tonka beans or Calophyllum inophyllum), root (e.g., in Ferulago campestris), and leaf (e.g., Murraya paniculata) [1]. All coumarins have a hydroxy or methoxy group at position 7. Scopoletin, esculetin, umbelliferone, fraxetin, as well as their respective glycosides, are termed simple coumarins; they are widespread in higher plants [2]. These coumarins play a pivotal role in protecting plants against pathogens [3]; furthermore, a simple coumarin, such as fraxetin, was found to modulate vital physiological processes such as iron homeostasis [4]. As naturally occurring phytochemicals, coumarins possess health-enhancing properties,

\footnotetext{
*Correspondence: jhahn@konkuk.ac.kr

Department of Bioscience and Biotechnology, Bio/Molecular Informatics Center, Konkuk University, Seoul 05029, Republic of Korea
}

including anticancer [1], neuroprotective [5], and antibacterial properties [6].

Coumarins were synthesized from hydroxycinnamic acids, such as $p$-coumaric acid, caffeic acid, and ferulic acid, in plants; $p$-coumaric acid, caffeic acid, and ferulic acid resulted in the synthesis of umbelliferone, esculetin, and scopoletin, respectively. The key enzyme for coumarin biosynthesis was $p$-coumaroyl CoA $2^{\prime}$-hydroxylase $\left(\mathrm{C}^{\prime} \mathrm{H}\right)$ or feruloyl CoA $6^{\prime}$-hydroxylase $\left(\mathrm{F} 6^{\prime} \mathrm{H}\right)$; the corresponding genes were cloned in Arabidopsis thaliana [7], Ruta graveolens [8], Ipomoea batatas [9], Manihot esculenta [10], Angelica decursiva [11], and Peucedanum praeruptorum [12]. This enzyme is a 2-oxglutarate-dependent dioxygenase; the hydroxylation of hydroxycinnamoyl-CoA resulted in the formation of a pyrone ring.

Fraxetin belongs to the family of simple coumarins and is synthesized from scopoletin by the hydroxylation of its carbon at position 8. Fraxetin is involved in iron metabolism in plants $[4,13]$. Similar to other phytochemicals, fraxetin was found to exert beneficial effects in humans. These included antitumor [10, 14], neuroprotective [15], 
antihyperglycemic [16], and anti-inflammatory [17] effects.

Since the metabolic pathway responsible for the synthesis of simple coumarins is well established, these compounds have been synthesized in E. coli. Scopoletin, esculetin, umbelliferone, skimming (umbelliferone 7-O-glucoside), and herniarin (7-O-methyl umbelliferone) were synthesized in E. coli $[18,19]$. For the fraxetin synthesis process in $E$. coli, two routes were postulated (Fig. 1). The first route involved the synthesis of esculetin from glucose, followed by the conversion of esculetin into fraxetin by 7-O-methylation and 8-hydroxylation. The second route started with the synthesis of scopoletin from ferulic acid, followed by 8-hydroxylation. In the present study, one coumarin, namely fraxetin, was synthesized using $E$. coli via these two routes.

\section{Materials and methods}

\section{Plasmid construction}

Reverse transcription polymerase chain reaction (RTPCR) was used to clone cDNA of scopoletin 8-hydroxylase (S8H) from Arabidopsis thaliana (AtS8H; GenBank: DQ446658.1). Two primers 5'-aagaattcaATGGGTATC AATTTCGAGGA-3' and 5'-aagcggccgcTCACTCGGC ACGTG-3' were used (restriction sites for EcoRI and NotI have been underlined). Additionally, the $S 8 H$ homologue from Oryza sativa (OsS8H; GenBank: XM_026024461) was cloned by RT-PCR using two primers: 5'-aagaattcaATGCCGTCCGGCTACGAC-3' and 5 '-aagcggccgcCTAATCTAGACTAGCGGCGG-3' (restriction sites for EcoRI and NotI have been underlined). AtS8H was digested using the EcoRI and NotI sites and subcloned into pGEX 5X-3 (pG-AtS8H), pET-duet1 (pE-AtS8H), pRSF-duet1 (pR-AtS8H), and pCDF-duet1
(pC-AtS8H). OsS8H was subcloned into EcoRI/NotI sites of pGEX 5X-3 (pG-OsS8H).

F6'H2 from Ipomoea batatas (IbF6'H2; GenBank : AB636154) and 4CL (Os4CL; 4-coumarate: CoA ligase) from $O$. sativa had been previously cloned using RT-PCR [18]. F6'H2 was first cloned into pET-duet1 (EcoRI/NotI) using PCR and, then, Os4CL was subcloned into pETduet1 containing $\mathrm{Fb}^{\prime} \mathrm{H} 2$ to generate $\mathrm{pE}-\mathrm{IbF} 6^{\prime} \mathrm{H} 2-\mathrm{Os} 4 \mathrm{CL}$ (NdeI/XhoI). Subsequently, Os4CL was re-amplified with a forward primer, adding a NotI site and ribosomal-binding site (RBS), and a reverse primer, containing a $X h o I$ site. Thereafter, $O s 4 C L$ was subcloned into the NotI/XhoI sites of pET-duet1 containing $I b F 6^{\prime} H 2$ to generate $\mathrm{pE}-\mathrm{IbF} 6^{\prime} \mathrm{H}-\mathrm{Os} 4 \mathrm{CL}$ controlled by a single promoter (operon). The IbF6 ${ }^{\prime} \mathrm{H}-\mathrm{Os} 4 \mathrm{CL}$ operon was subcloned into pGEX 5X-3 (EcoRI/XhoI).

POMT7 (flavone 7-O-methyltransferase) [20] and POMT9 from Populus deltoids [21] and ROMT9 (flavonoid 3'-O-methyltransferase) from O. sativa [22] have also been cloned previously. These genes were subcloned into pGEX 5X-3 vector.

\section{Production and analysis of metabolites}

For the synthesis of fraxetin from scopoletin, an overnight culture of an $E$. coli transformant containing pG-OsS8H, pG-AtS8H, pC-AtS8H, pE-AtS8H, or pRAtS8H was inoculated into fresh LB medium containing $50 \mu \mathrm{g} / \mathrm{mL}$ ampicillin and grown at $37^{\circ} \mathrm{C}$ until the $\mathrm{OD}_{600}$ reached 0.8 ; following this, isopropyl $\beta$-D-1thiogalactopyranoside (IPTG) was added to the medium at a final concentration of $0.1 \mathrm{mM}$ or $1 \mathrm{mM}$ and incubated at $18{ }^{\circ} \mathrm{C}$ for $16 \mathrm{~h}$. Cells were harvested and the cell concentration was adjusted to an $\mathrm{OD}_{600}$ of 3.0. The cells were resuspended in M9 medium containing $2 \%$ glucose, ampicillin $(50 \mu \mathrm{g} / \mathrm{mL})$, and either $0.1 \mathrm{mM}$ or $1 \mathrm{mM}$ IPTG

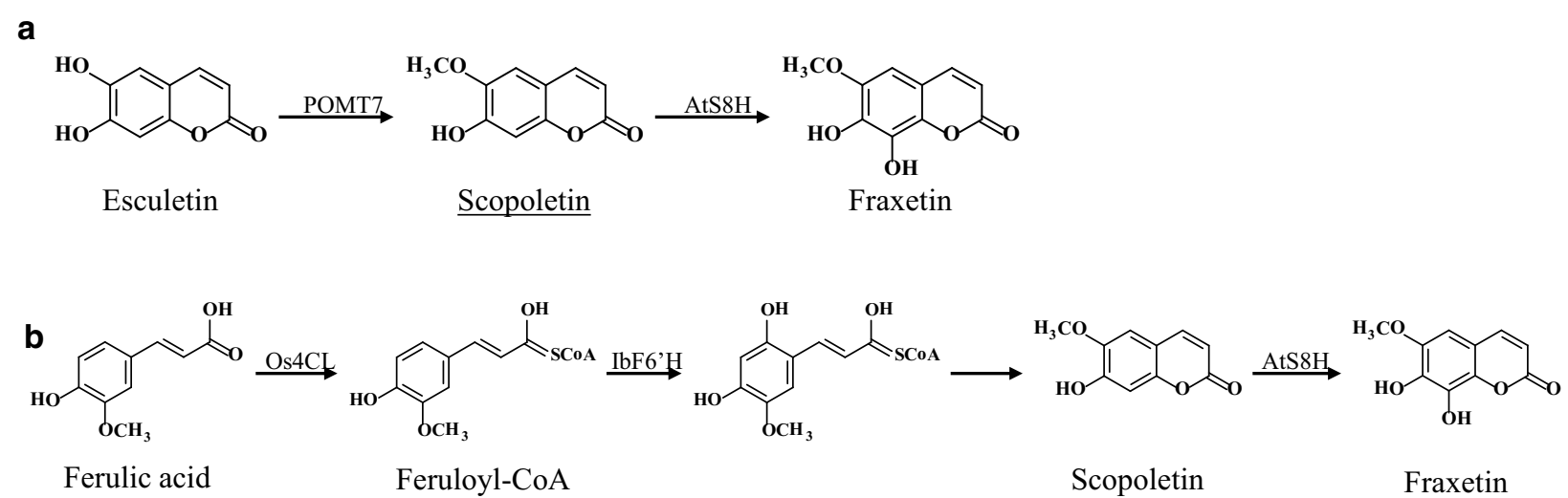

Fig. 1 Biosynthetic pathways of fraxetin from esculetin (a) and ferulic acid (b). POMT7 is an O-methyltransferase, which converts esculetin into scopoletin. AtS8H is a scopoletin 8-hydroxylase. Os4CL catalyzes the attachment of CoA to ferulic acid. IbF6' H encodes feruloyl CoA 6'-hydroxylase $\left(\mathrm{F} 6^{\prime} \mathrm{H}\right)$, which converts feruloyl CoA into scopoletin 
in a test tube. A total concentration of $100 \mu \mathrm{M}$ of the substrate (esculetin, isoscopoletin, scopoletin, or scoparone) was added, and the resulting culture was incubated at $30{ }^{\circ} \mathrm{C}$ for $24 \mathrm{~h}$. An E. coli transformant containing the pG-AtS8H construct was employed to determine the substrate (scopoletin) concentration. The cell concentration was adjusted to an $\mathrm{OD}_{600}$ of 3.0. The substrate was added to the appropriate M9 medium at $0.1,0.2,0.3$, or $0.5 \mathrm{mM}$. The reaction culture was incubated at $30{ }^{\circ} \mathrm{C}$ for $24 \mathrm{~h}$.

The E. coli transformant harboring ROMT9 was used to methylate esculetin to scopoletin, isoscopoletin, and scopoletin. Three reaction products were purified using thin layer chromatography (silica gel 60 F254, Millipore). A mixture of benzene and ethyl acetate (3:1) was used as a solvent. The E. coli transformant harboring POMT9 was used to synthesize scopoletin from esculetin. The methylation reaction using $E$. coli was carried out as described by Kim et al. [20].

Analysis of the reaction products was carried out using Thermo Ultimate 3000 HPLC [23]. Mass spectrometry and proton nuclear magnetic resonance (NMR) were performed as previously described $[24,25]$. The ${ }^{1} \mathrm{H}$ NMR of fraxetin in acetone- $\mathrm{d}_{6}$ (in ppm) is $\delta 3.87\left(3 \mathrm{H}, \mathrm{s}, 6-\mathrm{OCH}_{3}\right)$,
$6.15(1 \mathrm{H}, \mathrm{d}, J=9.3 \mathrm{~Hz}, \mathrm{H}-3), 6.76(1 \mathrm{H}, \mathrm{s}, \mathrm{H}-5), 7.91(1 \mathrm{H}$, d, $J=9.3 \mathrm{~Hz}, \mathrm{H}-4)$ [26].

\section{Results and discussion}

Biotransformation of scopoletin into fraxetin using $E$. coli harboring scopoletin 8-hydroxylase

Fraxetin is 8-hydroxy scopoletin. $S 8 H$ from $A$. thaliana $(\mathrm{AtS} 8 H)$ and its homologue from rice $(\mathrm{OsS} 8 H)$ were cloned as a glutathione $S$-transferase fusion protein and expressed in E. coli. Scopoletin was tested, along with other structurally-related coumarins, such as esculetin, isoscopoletin, and scoparone. These four compounds have esculetin derivatives. Three methylated esculetins (isoscopoletin, scopoletin, and scoparone) were synthesized using E. coli harboring ROMT9, purified, and used as substrates.

E. coli harboring AtS8H or $\mathrm{OsS} 8 \mathrm{H}$ was tested for the conversion of esculetin, isoscopoletin, scopoletin, and scoparone by the administration of each compound. $E$. coli harboring $\mathrm{OsS} 8 \mathrm{H}$ did not convert any coumarins used. However, for $E$. coli harboring AtS8H, scopoletin and isoscopoletin were converted into novel compounds that had retention times different from those of the corresponding substrates (Fig. 2). Other substrates

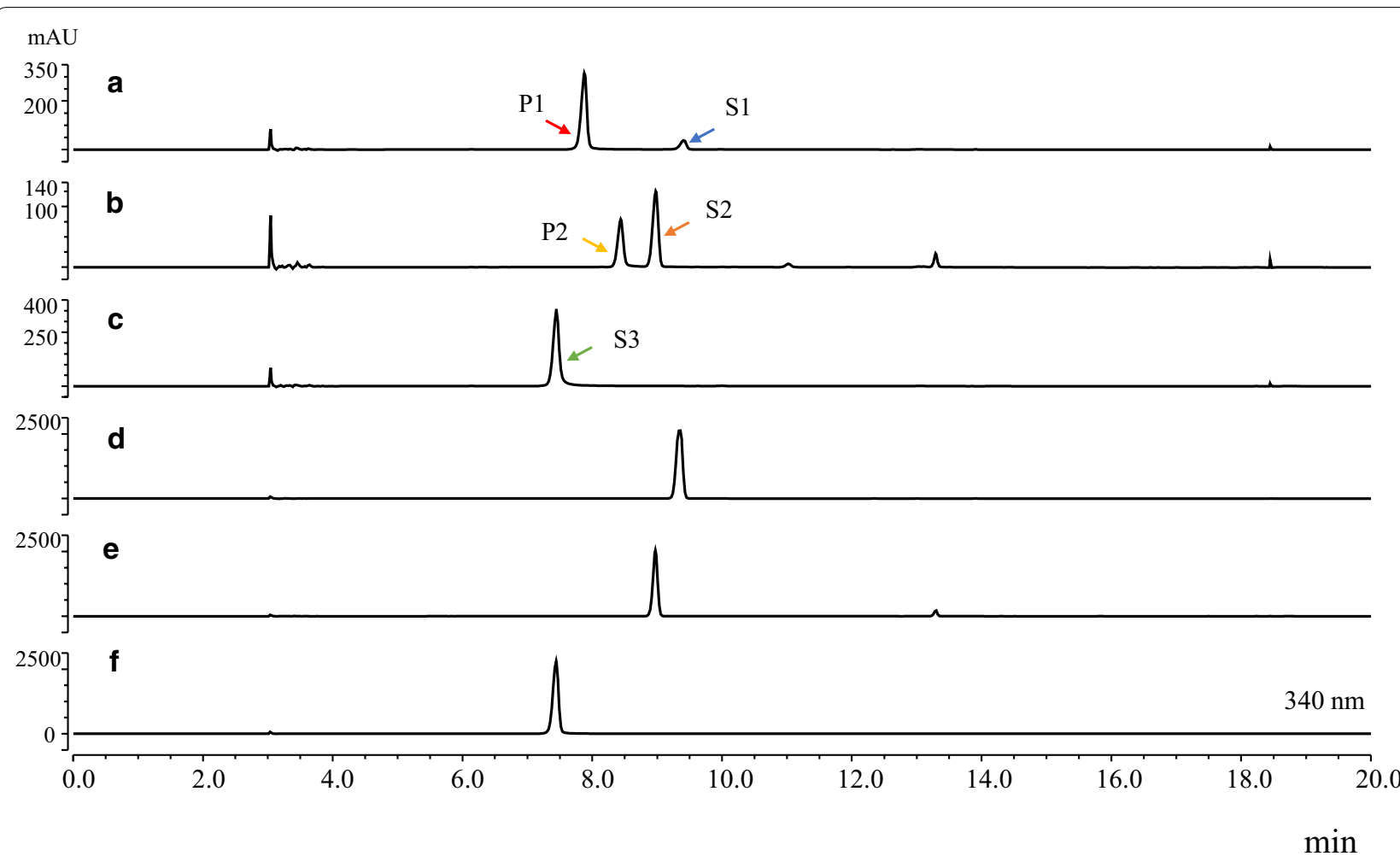

Fig. $2 \mathrm{HPLC}$ analysis of the reaction in E. coli harboring AtS8H. E. coli harboring AtS8H was administered scopoletin (a), isoscopoletin (b), and esculetin (c); the reaction product was analyzed. $\mathbf{d}-\mathbf{f}$ denote standard scopoletin, isoscopoletin, and esculetin, respectively. P1, reaction product from scopoletin; P2, reaction product from isoscopoletin; S1, scopletin; S2, isoscopoletin; S3, esculetin 
(esculetin and scoparone) did not generate any new product. The molecular mass of the products from scopoletin and isoscopoletin was $207.937 \mathrm{Da}$, which is the molecular mass obtained if hydroxylation occurs. S8H utilized one methylated esculetins (scopoletin and isoscopoletin) as a substrate and did not utilize dimethylated (scoparone) or unmethylated esculetin. Scopoletin was a better substrate than isoscopoletin; $84.8 \%$ of scopoletin was converted, as opposed to the conversion of only $55 \%$ isoscopoletin. To determine the structure of the biotransformation product from scopoletin, the reaction product was purified, and its structure was analyzed using proton NMR. The reaction product was determined to be fraxetin (see Materials and Methods). E. coli harboring different constructs (pGAtS8H, pE-AtS8H, pR-AtS8H, or pC-AtS8H) synthesized the approximately same amount fraxetin from scopoletin.

To optimize the initial concentration of scopoletin and the final yield of fraxetin, E. coli harboring $A t S 8 H$ was prepared at an $\mathrm{OD}_{600}$ of 3.0 after induction of AtS8H. Subsequently, four different concentrations of scopoletin $(100,200,300$, and $500 \mu \mathrm{M})$ were added. The highest rate of conversion of scopoletin into fraxetin was seen at $100 \mu \mathrm{M}$ scopoletin; $84.8 \mu \mathrm{M}$ fraxetin was synthesized (84.8\% conversion rate). However, fraxetin production was highest at $200 \mu \mathrm{M}$ scopoletin; approximately $139.5 \mu \mathrm{M}$ fraxetin was synthesized $(69.8 \%$ conversion rate). Above $200 \mu \mathrm{M}$ scopoletin, the production level of fraxetin registered a decline. The optimum initial cell concentrations were also determined. Five initial cell concentrations $\left(\mathrm{OD}_{600}=1.0,2.0,3.0,4.0\right.$, and 5.0) were tested and $200 \mu \mathrm{M}$ scopoletin was administered. As the initial cell concentration increased, the conversion of scopoletin also registered a concomitant increase. At an $\mathrm{OD}_{600}$ of 5.0, approximately $152.0 \mu \mathrm{M}$ of scopoletin was converted into fraxetin.

\section{Synthesis of fraxetin from esculetin and ferulic acid}

Fraxetin may also be synthesized from esculetin. Two enzymatic reactions are required; the first is the conversion of esculetin into scopoletin by an $O$-methyltransferase (OMT), and the second is the synthesis of fraxetin from scopoletin by $\mathrm{S} 8 \mathrm{H}$. For the synthesis of scopoletin from esculetin, three OMT genes (POMT7, POMT9, and ROMT9) were evaluated. E. coli harboring POMT7 synthesized $56.8 \mu \mathrm{M}$ scopoletin from $100 \mu \mathrm{M}$ esculetin (Fig. 3a). However, E. coli harboring ROMT9 produced three methylated esculetins (isoscopoletin, scopoletin, and scoparone), with isoscopoletin as a major product. The ratio of isoscopoletin, scopoletin, and scoparone was 83: 13: 3. POMT9 generated almost the same amounts of isoscopoletin $(38.1 \mu \mathrm{M})$ and scopoletin $(37.4 \mu \mathrm{M})$ from $100 \mu \mathrm{M}$ esculetin. Therefore, E. coli harboring POMT7 was utilized to synthesize scopoletin from esculetin.

A two-step reaction was conducted using two E. coli transformants to augment the final yield of fraxetin. The first reaction was carried out using $E$. coli harboring POMT7. Approximately $56.9 \mu \mathrm{M}$ scopoletin was synthesized from $100 \mu \mathrm{M}$ esculetin (Fig. 3a). Further incubation did not result in the conversion of more esculetin. Thereafter, the culture filtrate from the first reaction was combined with $E$. coli harboring AtS8H. Approximately $41.4 \mu \mathrm{M}$ fraxetin was synthesized from $56.9 \mu \mathrm{M}$ scopoletin (Fig. 3b), indicating that there was approximately $72.7 \%$ conversion from the synthesized fraxetin.

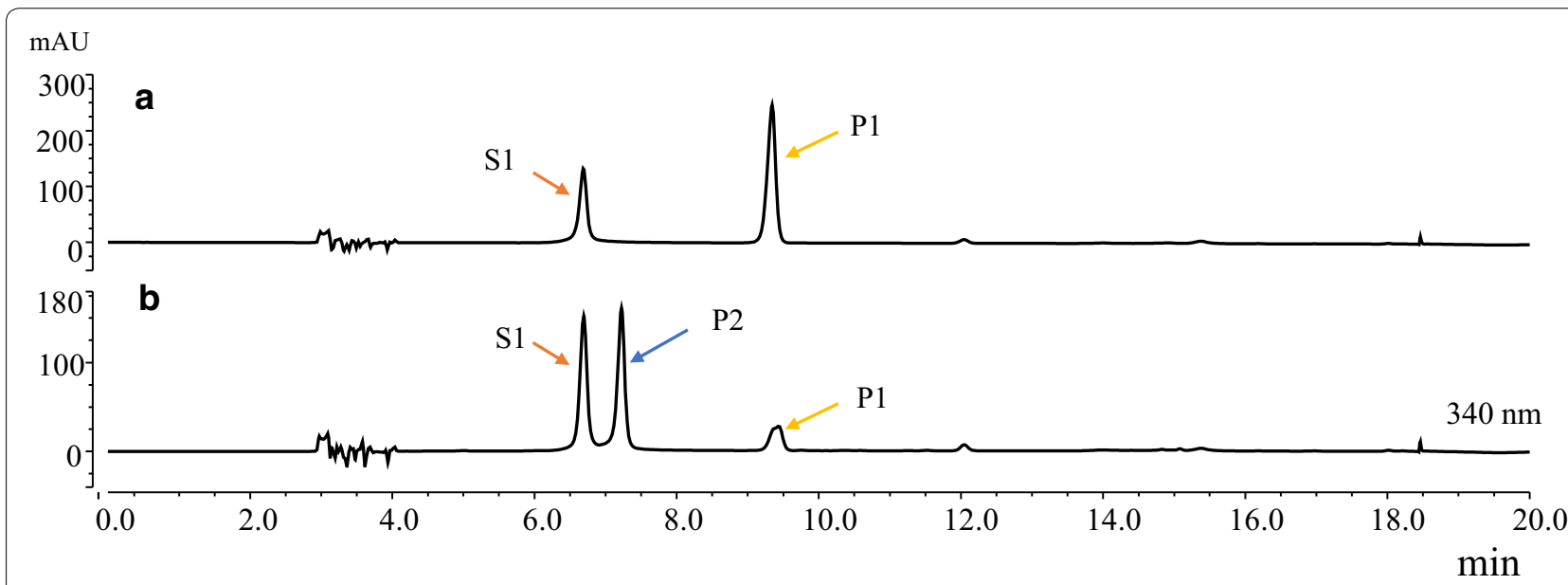

Fig. 3 Synthesis of fraxetin from esculetin using two E. coli transformants. a Conversion of esculetin into scopoletin using E. coli harboring POMT7. E. coli harboring POMT7 was administered esculetin (S1), following which the reaction product was analyzed. P1 denotes the reaction product from scopoletin. $\mathbf{b}$ Synthesis of fraxetin from scopoletin using E. coli harboring AtS8H. The culture filtrate from E. coli harboring POMT7 was administered to the E. coli harboring AtSBH, and the reaction product was analyzed 


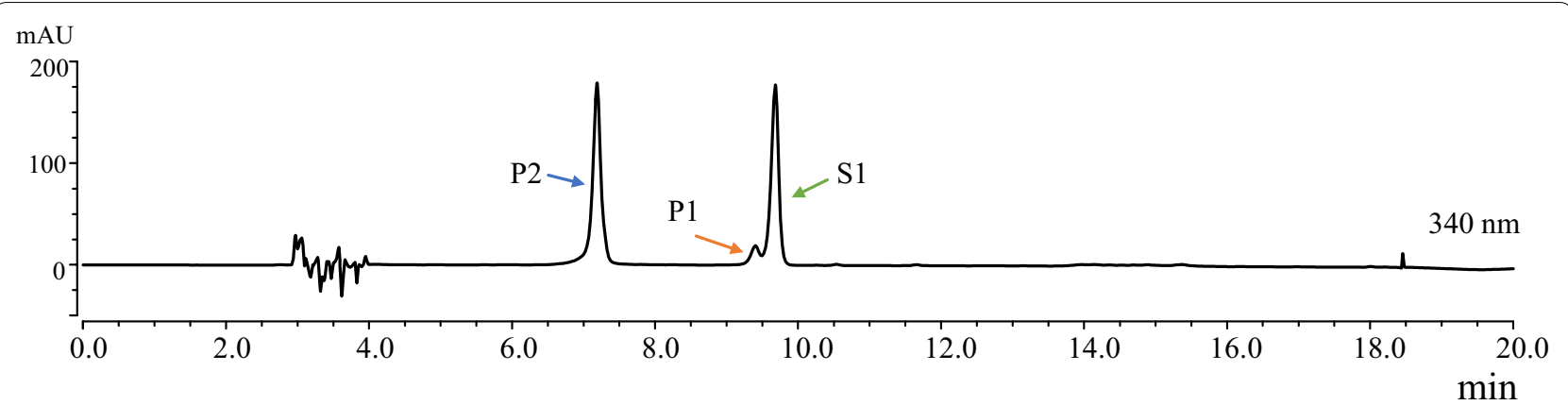

Fig. 4 Synthesis of fraxetin from ferulic acid using E. coli harboring Os4CL, lbF6' H, and AtS8H. S1, ferulic acid; P1, scopoletin; P2, fraxetin

Fraxetin was successfully synthesized from esculetin by a two-step reaction. The final yield of fraxetin synthesized from esculetin was lower than that from scopoletin; moreover, the conversion rate of scopoletin into fraxetin in the two-step reaction was lower than that seen for the direct conversion. This could possibly be attributed to the metabolite(s) in the first step inhibiting the second reaction. It was attempted herein to synthesize fraxetin from esculetin using an $E$. coli transformant harboring both POMT7 and AtS8H. Only $3.4 \mu \mathrm{M}$ fraxetin and $17.2 \mu \mathrm{M}$ scopoletin were synthesized from $100 \mu \mathrm{M}$ esculetin.

Next, fraxetin was synthesized from ferulic acid. Three enzymatic reactions are required for this. Previously, scopoletin was successfully synthesized from ferulic acid using E. coli harboring $I b F 6^{\prime} H 2$ and Os4CL. It was reasoned that introducing AtS8H into E. coli harboring $I b F \sigma^{\prime} H 2$ and $O s 4 C L$ could result in the synthesis of fraxetin from ferulic acid. Three genes $\left(I b F 6^{\prime} H, O s 4 C L\right.$, and $A t S 8 H)$ were introduced into $E$. coli, and the resulting transformant was administered ferulic acid. The $E$. coli transformant synthesized fraxetin from ferulic acid. To optimize fraxetin synthesis, several initial ferulic acid concentrations $(100,200,300$, and $500 \mu \mathrm{M})$ were tested. The synthesis of fraxetin was optimal at $100 \mu \mathrm{M}$ of initial ferulic acid, and approximately $33.3 \mu \mathrm{M}$ fraxetin was synthesized (Fig. 4). Unreacted ferulic acid and scopoletin were accumulated at the higher concentrations of ferulic acid.

Fraxetin was synthesized from three different substrates (scopoletin, esculetin, and ferulic acid). As shown in Fig. 1, more enzymes are required when fraxetin is synthesized from ferulic acid or esculetin than when it is synthesized from scopoletin. Consequently, the final yield of fraxetin was higher $(84.8 \mu \mathrm{M})$ when it was synthesized from scopoletin $(100 \mu \mathrm{M})$. Its yield was decreased when synthesis was carried out from esculetin $(41.4 \mu \mathrm{M})$ or ferulic acid $(33.3 \mu \mathrm{M})$.

An attempt was made to synthesize fraxetin from esculetin or ferulic acid. One E. coli transformant harboring both POMT7 and AtS8H synthesized a lower amount of fraxetin from esculetin than the other two $E$. coli transformants, each of which conducted one reaction. However, fraxetin was successfully synthesized from ferulic acid using one $E$. coli transformant harboring three genes (Os4CL, IbF6' $H$, and $A t S 8 H)$. Esculetin may compete with scopoletin for AtS8H. In the E. coli transformant harboring both POMT7 and AtS8H, esculetin served as a substrate for POMT7 and an inhibitor of AtS8H. Therefore, following the synthesis of scopoletin by POMT7, AtS8H could not utilize scopoletin because it was inhibited by esculetin. Conversely, when two independent $E$. coli transformants were used, more scopoletin synthesized by the first $E$. coli transformant harboring POMT7 was present in the medium and was converted into fraxetin by the second E. coli transformant harboring AtS8H. When fraxetin was synthesized from ferulic acid, only scopoletin was synthesized; therefore, it was possible to synthesize fraxetin using $E$. coli harboring $O s 4 C L, I b F 6^{\prime} H$, and $A t S 8 H$.

\section{Acknowledgements}

The present study was supported by grants from the Next-Generation BioGreen 21 Program (PJ01326001), Rural Development Administration, Republic of Korea.

\section{Authors' contributions}

SHA and JHA designed the experiments. SHA, GSC, and JHA performed the experiments and analyzed the data. SHA, GSC, and JHA wrote the manuscript. All authors read and approved the final manuscript.

\section{Funding}

Funding was received from the Next-Generation BioGreen 21 Program, Rural Development Administration (PJ01326001).

\section{Availability of data and materials}

All data generated or analyzed during the present study are included in this published article.

\section{Competing interests}

The authors declare that they have no competing interests.

Received: 5 August 2020 Accepted: 9 September 2020

Published online: 14 September 2020 


\section{References}

1. Thakur A, Singla R, Jaitak V (2015) Coumarins as anticancer agents: a review on synthetic strategies, mechanism of action and SAR studies. Eur J Med Chem 101:476-495

2. Bourgaud F, Hehn A, Larbat R, Doerper S, Gontier E, Kellner S, Matern U (2006) Biosynthesis of coumarins in plants: a major pathway still to be unraveled for cytochrome P450 enzymes. Phytochem Rev 5:293-308

3. Stringlis IA, de Jonge R, Pieterse CMJ (2019) The age of coumarins in plant-microbe interactions. Plant Cell Physiol 60:1405-1419

4. Tsai HH, Rodríguez-Celma J, Lan P, Wu YC, Vélez-Bermúdez IC, Schmidt W (2018) Scopoletin 8-hydroxylase-mediated fraxetin production is crucial for iron mobilization. Plant Physiol 177:194-207

5. Kang SY, Kim YC (2007) Neuroprotective coumarins from the root of Angelica gigas: structure-activity relationships. Arch Pharm Res 30:1368-1373

6. Kayser O, Kolodziej H (1999) Antibacterial activity of simple coumarins: structural requirements for biological activity. Z Naturforsch, C: J Biosci 54:169-174

7. Kai K, Mizutani M, Kawamura N, Yamamoto R, Tamai M, Yamaguchi H, Sakata K, Schimizu B-I (2008) Scopoletin is biosynthesized via orthohydroxylation of feruloyl CoA by a 2-oxoglutarate-dependent dioxygenase in Arabidopsis thaliana. Plant J 55:989-999

8. Vialart G, Hehn A, Olry A, Ito K, Krieger C, Larbat R, Paris C, Bun-Ichi S, Sugimoto Y, Mizutani M, Bourgaud F (2012) A 2-oxoglutarate dependent dioxygenase from Ruta graveolens $L$. exhibits $p$-coumaroyl CoA $2^{\prime}$ hydroxylase activity $\left(\mathrm{C}^{\prime} \mathrm{H}\right)$ : a missing step in the synthesis of umbelliferone. in plants. Plant J 70:460-470

9. Matsumoto S, Mizutani M, Sakata K, Shimizu B (2012) Molecular cloning and functional analysis of the ortho-hydroxylases of $p$-coumaroyl coenzyme A/feruloyl coenzyme A involved in formation of umbelliferone and scopoletin in sweet potato, Ipomoea batatas (L.) Lam. Phytochemistry 74:49-57

10. Liu S, Zainuddin IM, Vanderschuren H, Doughty J, Beeching JR (2017) RNAi inhibition of feruloyl CoA 6'-hydroxylase reduces scopoletin biosynthesis and post-harvest physiological deterioration in cassava (Manihot esculenta (rantz) storage roots. Plant Mol Biol 94:185-195

11. Zhao Y, Jian X, Wu J, Huang W, Huang C, Luo J, Kong L (2019) Elucidation of the biosynthesis pathway and heterologous construction of a sustainable route for producing umbelliferone. J Biol Eng 13:44

12. Yao R, Zhao Y, Liu T, Huang C, Xu S, Sui Z, Luo J, Kong L (2017) Identification and functional characterization of a $p$-coumaroyl CoA 2 '-hydroxylase involved in the biosynthesis of coumarin skeleton from Peucedanum praeruptorum Dunn. Plant Mol Biol 95:199-213

13. Siwinska J, Siatkowska K, Olry A, Grosjean J, Hehn A, Bourgaud F, Meharg AA, Carey M, Lojkowska E, Ihnatowicz A (2018) Scopoletin 8-hydroxylase: a novel enzyme involved in coumarin biosynthesis and iron-deficiency responses in Arabidopsis. J Exp Bot 69:1735-1748

14. Kimura Y, Sumiyoshi M (2015) Antitumor and antimetastatic actions of dihydroxycoumarins (esculetin or fraxetin) through the inhibition of M2 macrophage differentiation in tumor-associated macrophages and/or G1 arrest in tumor cells. Eur J Pharm 746:115-125

15. Molina-Jiménez MF, Sánchez-Reus MI, Andres D, Cascales M, Benedi J (2004) Neuroprotective effect of fraxetin and myricetin against rotenoneinduced apoptosis in neuroblastoma cells. Brain Res 1009:9-16

16. Murali R, Srinivasan S, Ashokkumar N (2013) Antihyperglycemic effect of fraxetin on hepatic key enzymes of carbohydrate metabolism in streptozotocin-induced diabetic rats. Biochimie 95:1848-1854

17. Kundu J, Chae IG, Chun K-S (2016) Fraxetin induces heme oxygenase-1 expression by activation of Akt/Nrf2 or AMP-activated protein kinase a/ Nrf2 pathway in HaCaT cells. J Cancer Prev 21:135-143

18. Yang S-M, Shim GY, Kim B-G, Ahn J-H (2015) Biological synthesis of coumarins in Escherichia coli. Microb Cell Fact 14:65

19. Chu LL, Pandey RP, Lim HN, Jung HJ, Thuan NH, Kim T-S, Sohng JK (2017) Synthesis of umbelliferone derivatives in Escherichia coli and their biological activities. J Biol Eng 11:15

20. Kim BG, Lee YJ, Lee S, Lim Y, Cheong Y, Ahn J-H (2008) Altered regioselectivity of a poplar O-methyltransferase, POMT-7. J Biotech 138:107-111

21. Kim BG, Lee Y, Hur HG, Lim Y, Ahn J-H (2006) Production of Three O-Methhylated Esculetins with E. coli Expressing O-Methyltransferase from Poplar. Biosci Biotech Biochem 70:1269-1272

22. Kim BG, Lee Y, Hur H-G, Lim Y, Ahn J-H (2006) Flavonoid 3'-O-methyltransferase from rice: cDNA cloning, characterization and functional expression. Phytochemistry 67:387-394

23. Lee SJ, Sim GY, Kang H, Yeo WS, Kim B-G, Ahn J-H (2018) Synthesis of avenanthramides using engineered Escherichia coli. Microb Cell Fact 17:46

24. Song MK, Cho AR, Sim GY, Ahn J-H (2019) Synthesis of diverse hydroxycinnamoyl phenylethanoid esters using Escherichia coli. J Agric Food Chem 67:2028-2035

25. Yoon J-A, Kim B-G, Lee WJ, Lim Y, Chong Y, Ahn J-H (2012) Production of a novel quercetin glycoside through metabolic engineering of Escherichia coli. Appl Env Microbiol. 78:4256-4262

26. Yu M, Sun A, Zhnag Y, Liu R (2014) Purification of coumarin compounds from Cortex fraxinus by adsorption chromatography. J Chromatogr Sci 52:1033-1037

\section{Publisher's Note}

Springer Nature remains neutral with regard to jurisdictional claims in published maps and institutional affiliations.

\section{Submit your manuscript to a SpringerOpen ${ }^{\circ}$ journal and benefit from:}

- Convenient online submission

- Rigorous peer review

- Open access: articles freely available online

- High visibility within the field

Retaining the copyright to your article

Submit your next manuscript at springeropen.com 